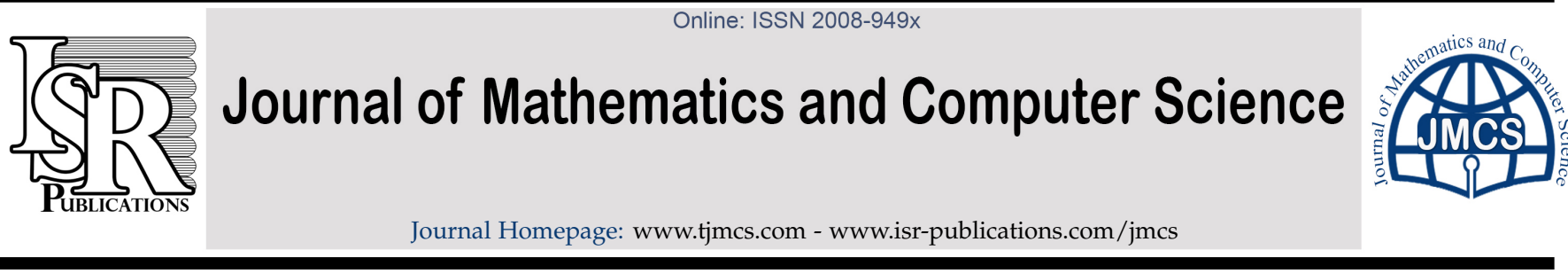

\title{
4-step 5-point hybrid block method for the direct solution of second order initial value problems
}

\author{
Adeyeye Oluwaseun*, Omar Zurni \\ School of Quantitative Sciences, Universiti Utara Malaysia, Sintok, Kedah, Malaysia.
}

\begin{abstract}
Block methods for the numerical solution of ordinary differential equations (ODEs) are quite prominent in recent literature and second order initial value problems (IVPs) which falls in the family of ODEs is also a well explored area for the application of block methods. The introduction of hybrid block method methods for the solution of second order IVPs has gained good grounds in literature as the presence of off-grid points in the block method has increased the accuracy of the hybrid block methods. However, recent studies still continue to introduce new block methods that will perform more favourably than previously existing when compared in terms of error. Hence, a hybrid block method of order six is presented in this article to compete with previously existing methods of the same order and higher order. The methodology adopted in this article presents a new approach for developing the hybrid block method which is simple to implement and less computationally tiresome. The numerical results show this new 4-step 5-point hybrid block method performing better than previously existing methods. (C) 2017 All rights reserved.
\end{abstract}

Keywords: Hybrid, block method, order six, second order, initial value problems. 2010 MSC: 65L05, 65L06.

\section{Introduction}

Consider the following equation

$$
y^{\prime \prime}=f\left(x, y, y^{\prime}\right), \quad y\left(x_{0}\right)=y_{0}, y^{\prime}\left(x_{0}\right)=y_{0}^{\prime} .
$$

It is of general knowledge that equations in the form (1.1) above with such conditions imposed are known as initial value problems (IVPs). Numerical solution of equations of this form is a very keen area of interest for researchers in literature, ranging from first order IVPs $[6,11]$ to higher order IVPs $[7,13]$. The focus of this article however is on higher order IVPs (specifically, second order IVPs). Block methods for approximating the numerical solution of (1.1) have been vastly explored in literature. The key advantage of the adoption of block method has been in the improved level of accuracy displayed in comparison to previously existing methods (see $[4,9,10]$ ).

\footnotetext{
*Corresponding author

Email address: adeyeye_oluwaseun@ahsgs .uum.edu.my (Adeyeye Oluwaseun)
} 
Hybrid block methods on the other hand, which involve considering off-grid points when developing the block method have also been seen to be very effective when solving (1.1) in literature. The application of hybrid methods for the numerical approximation of (1.1) has been applied in predictor-corrector modes [8], block-predictor corrector [2] and direct block methods [3, 4]. Although, this approach involves more function steps computation, the results obtained when solving numerical problems shows great advantage, especially when in comparison to previous methods of the same order or higher order as displayed in this article. This article is interested in considering recent non-hybrid block methods in literature of order six and higher that have been adopted to approximate (1.1). Order six was chosen as the developed hybrid block method in this work is of order six, hence a good basis for comparing the numerical results. These numerical results, as displayed at the latter end of this article has shown good superiority of the new hybrid block method when solving (1.1).

Likewise, the most commonly used approach for developing block methods has been the adoption of the approach seen in [1]. This is seen recent works of $[6,9,14]$. However, this article introduces another approach different from what is existing in literature on how hybrid block methods can also be developed. Therefore, asides from the introduction of a new hybrid block method that performs better than previously existing methods, this article also introduces a new approach for the development of hybrid block methods.

\section{Derivation of the method}

Consider the following multistep scheme

$$
y_{n+\frac{4}{5} \xi}=\sum_{i=0}^{1} \frac{\left(\frac{4}{5} \xi h\right)^{i}}{i !} y_{n}^{(i)}+h^{2} \sum_{i=0}^{5} \phi_{\xi i} f_{n+\frac{4}{5} i}, \quad \xi=1,2, \cdots, 5,
$$

where its corresponding first derivative scheme is obtained from

$$
y_{n+\frac{4}{5} \xi}^{\prime}=y_{n}^{\prime}+h \sum_{i=0}^{5} \omega_{\xi i} f_{n+\frac{4}{5} i}, \quad \xi=1,2, \cdots, 5 .
$$

The unknown coefficients in (2.1) and (2.2) are evaluated by adopting matrix inverse approach as shown below, that is,

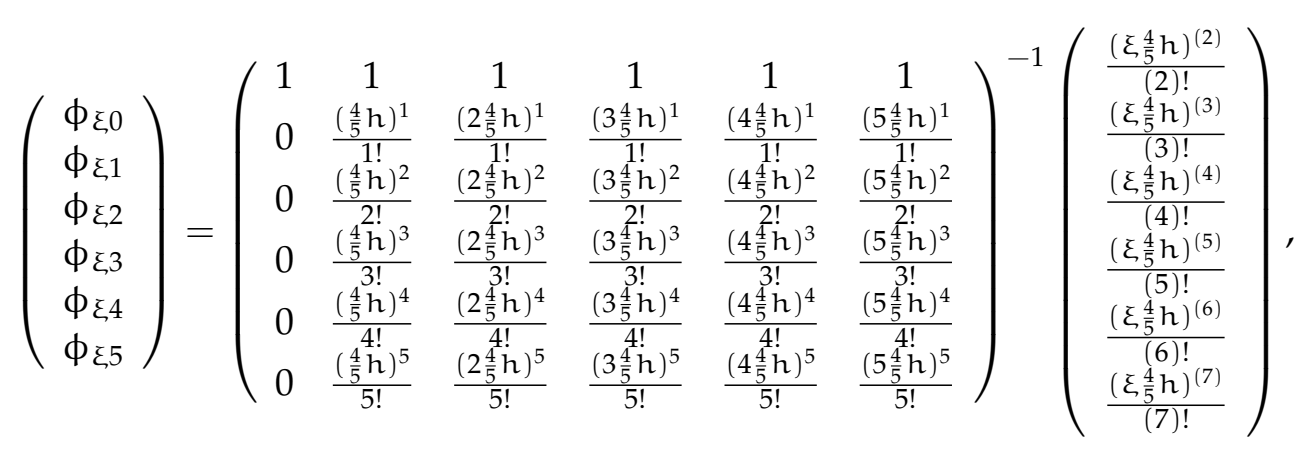

and

$$
\left(\begin{array}{l}
\omega_{\xi 0} \\
\omega_{\xi 1} \\
\omega_{\xi 2} \\
\omega_{\xi 3} \\
\omega_{\xi 4} \\
\omega_{\xi 5}
\end{array}\right)=\left(\begin{array}{cccccc}
1 & 1 & 1 & 1 & 1 & 1 \\
0 & \frac{\left(\frac{4}{5} h\right)^{1}}{1 !} & \frac{\left(2 \frac{4}{5} h\right)^{1}}{1 !} & \frac{\left(3 \frac{4}{5} h\right)^{1}}{1 !} & \frac{\left(4 \frac{4}{5} h\right)^{1}}{1 !} & \frac{\left(5 \frac{4}{5} h\right)^{1}}{1 !} \\
0 & \frac{\left(\frac{4}{5} h\right)^{2}}{2 !} & \frac{\left(2 \frac{4}{5} h\right)^{2}}{2 !} & \frac{\left(3 \frac{4}{5} h\right)^{2}}{2 !} & \frac{\left(4 \frac{4}{5} h\right)^{2}}{2 !} & \frac{\left(5 \frac{4}{5} h\right)^{2}}{2 !} \\
0 & \frac{\left(\frac{4}{5} h\right)^{3}}{3 !} & \frac{\left(\frac{2}{5} h\right)^{3}}{3 !} & \frac{\left(3 \frac{4}{5} h\right)^{3}}{3 !} & \frac{\left(4 \frac{4}{5} h\right)^{3}}{3 !} & \frac{\left(5 \frac{4}{5} h\right)^{3}}{3 !} \\
0 & \frac{\left(\frac{4}{5} h\right)^{4}}{4 !} & \frac{\left(2 \frac{4}{5} h\right)^{4}}{4 !} & \frac{\left(3 \frac{4}{5} h\right)^{4}}{4 !} & \frac{\left(4 \frac{4}{5} h\right)^{4}}{4 !} & \frac{\left(5 \frac{4}{5} h\right)^{4}}{4 !} \\
0 & \frac{\left(\frac{4}{5} h\right)^{5}}{5 !} & \frac{\left(2 \frac{4}{5} h\right)^{5}}{5 !} & \frac{\left(3 \frac{4}{5} h\right)^{5}}{5 !} & \frac{\left(4 \frac{4}{5} h\right)^{5}}{5 !} & \frac{\left(5 \frac{4}{5} h\right)^{5}}{5 !}
\end{array}\right)^{-1}\left(\begin{array}{c}
\frac{\left(\xi \frac{4}{5} h\right)^{(1)}}{(1) !} \\
\frac{\left(\xi \frac{4}{5} h\right)^{(2)}}{(2) !} \\
\left.\frac{(\xi 5)}{5} h\right)^{(3)} \\
\frac{\left(\xi \frac{4}{5} h\right)^{(4)}}{4(4) !} \\
\frac{\left(\xi \frac{4}{5} h\right)^{(5)}}{(5) !} \\
\frac{(\xi 5)}{(6) !}
\end{array}\right) .
$$


Hence, the coefficients are obtained as

$$
\begin{aligned}
& \left(\phi_{10}, \phi_{11}, \phi_{12}, \phi_{13}, \phi_{14}, \phi_{15}\right)^{\top}=\left(\frac{1231}{7875}, \frac{863}{3150},-\frac{1522}{7875}, \frac{941}{7875},-\frac{341}{7875}, \frac{107}{15750}\right) ; \\
& \left(\phi_{20}, \phi_{21}, \phi_{22}, \phi_{23}, \phi_{24}, \phi_{25}\right)^{\top}=\left(\frac{568}{1575}, \frac{8704}{7875},-\frac{592}{1575}, \frac{2176}{7875},-\frac{808}{7875}, \frac{128}{7875}\right) ; \\
& \left(\phi_{30}, \phi_{31}, \phi_{32}, \phi_{33}, \phi_{34}, \phi_{35}\right)^{\top}=\left(\frac{492}{875}, \frac{3501}{1750},-\frac{36}{875}, \frac{87}{175},-\frac{144}{875}, \frac{9}{350}\right) ; \\
& \left(\phi_{40}, \phi_{41}, \phi_{42}, \phi_{43}, \phi_{44}, \phi_{45}\right)^{\top}=\left(\frac{6016}{7875}, \frac{22784}{7875}, \frac{2816}{7875}, \frac{9728}{7875},-\frac{256}{1575}, \frac{256}{7875}\right) ; \\
& \left(\phi_{50}, \phi_{51}, \phi_{52}, \phi_{53}, \phi_{54}, \phi_{55}\right)^{\top}=\left(\frac{61}{63}, \frac{475}{126}, \frac{50}{63}, \frac{125}{63}, \frac{25}{63}, \frac{11}{126}\right) ; \\
& \left(\omega_{10}, \omega_{11}, \omega_{12}, \omega_{13}, \omega_{14}, \omega_{15}\right)^{\top}=\left(\frac{19}{72}, \frac{1427}{1800},-\frac{133}{300}, \frac{241}{900},-\frac{173}{1800}, \frac{3}{200}\right) ; \\
& \left(\omega_{20}, \omega_{21}, \omega_{22}, \omega_{23}, \omega_{24}, \omega_{25}\right)^{\top}=\left(\frac{56}{225}, \frac{86}{75}, \frac{28}{225}, \frac{28}{225},-\frac{4}{75}, \frac{2}{225}\right) ; \\
& \left(\omega_{30}, \omega_{31}, \omega_{32}, \omega_{33}, \omega_{34}, \omega_{35}\right)^{\top}=\left(\frac{51}{200}, \frac{219}{200}, \frac{57}{100}, \frac{57}{100},-\frac{21}{200}, \frac{3}{200}\right) ; \\
& \left(\omega_{40}, \omega_{41}, \omega_{42}, \omega_{43}, \omega_{44}, \omega_{45}\right)^{\top}=\left(\frac{56}{225}, \frac{256}{225}, \frac{32}{75}, \frac{256}{225}, \frac{56}{225}, 0\right) ; \\
& \left(\omega_{50}, \omega_{51}, \omega_{52}, \omega_{53}, \omega_{54}, \omega_{55}\right)^{\top}=\left(\frac{19}{72}, \frac{25}{24}, \frac{25}{36}, \frac{25}{36}, \frac{25}{24}, \frac{19}{72}\right) .
\end{aligned}
$$

Upon substitution of these coefficients back in (2.1) and (2.2) gives the following hybrid block schemes

$$
\begin{aligned}
y_{n+\frac{4}{5}}= & y_{n}+\frac{4}{5} h y_{n}^{\prime} \\
& +\frac{h^{2}}{15750}\left(2462 f_{n}+4315 f_{n+\frac{4}{5}}-3044 f_{n+\frac{8}{5}}+1882 f_{n+\frac{12}{5}}-682 f_{n+\frac{16}{5}}+107 f_{n+4}\right), \\
y_{n+\frac{8}{5}}= & y_{n}+\frac{8}{5} h y_{n}^{\prime} \\
& +\frac{h^{2}}{7875}\left(2840 f_{n}+8704 f_{n+\frac{4}{5}}-2960 f_{n+\frac{8}{5}}+2176 f_{n+\frac{12}{5}}-808 f_{n+\frac{16}{5}}+128 f_{n+4}\right), \\
y_{n+\frac{12}{5}}= & y_{n}+\frac{12}{5} h y_{n}^{\prime} \\
& +\frac{h^{2}}{1750}\left(984 f_{n}+3501 f_{n+\frac{4}{5}}-72 f_{n+\frac{8}{5}}+870 f_{n+\frac{12}{5}}-288 f_{n+\frac{16}{5}}+45 f_{n+4}\right), \\
y_{n+\frac{16}{5}}= & y_{n}+\frac{16}{5} h y_{n}^{\prime} \\
& +\frac{h^{2}}{7875}\left(6016 f_{n}+22784 f_{n+\frac{4}{5}}+2816 f_{n+\frac{8}{5}}+9728 f_{n+\frac{12}{5}}-1280 f_{n+\frac{16}{5}}+256 f_{n+4}\right), \\
y_{n+4}= & y_{n}+4 h y_{n}^{\prime} \\
& +\frac{h^{2}}{126}\left(122 f_{n}+475 f_{n+\frac{4}{5}}+100 f_{n+\frac{8}{5}}+250 f_{n+\frac{12}{5}}+50 f_{n+\frac{16}{5}}+11 f_{n+4}\right),
\end{aligned}
$$

with first derivative schemes given as

$$
\begin{aligned}
& y_{n+\frac{4}{5}}^{\prime}=y_{n}^{\prime}+\frac{h}{1800}\left(475 f_{n}+1427 f_{n+\frac{4}{5}}-798 f_{n+\frac{8}{5}}+482 f_{n+\frac{12}{5}}-173 f_{n+\frac{16}{5}}+27 f_{n+4}\right), \\
& y_{n+\frac{8}{5}}^{\prime}=y_{n}^{\prime}+\frac{h}{225}\left(56 f_{n}+258 f_{n+\frac{4}{5}}+28 f_{n+\frac{8}{5}}+28 f_{n+\frac{12}{5}}-12 f_{n+\frac{16}{5}}+2 f_{n+4}\right),
\end{aligned}
$$




$$
\begin{aligned}
& y_{n+\frac{12}{5}}^{\prime}=y_{n}^{\prime}+\frac{h}{200}\left(51 f_{n}+219 f_{n+\frac{4}{5}}+114 f_{n+\frac{8}{5}}+114 f_{n+\frac{12}{5}}-21 f_{n+\frac{16}{5}}+3 f_{n+4}\right), \\
& y_{n+\frac{16}{5}}^{\prime}=y_{n}^{\prime}+\frac{h}{225}\left(56 f_{n}+256 f_{n+\frac{4}{5}}+96 f_{n+\frac{8}{5}}+256 f_{n+\frac{12}{5}}+56 f_{n+\frac{16}{5}}\right), \\
& y_{n+4}^{\prime}=y_{n}^{\prime}+\frac{h}{72}\left(19 f_{n}+75 f_{n+\frac{4}{5}}+50 f_{n+\frac{8}{5}}+50 f_{n+\frac{12}{5}}+75 f_{n+\frac{16}{5}}+19 f_{n+4}\right) .
\end{aligned}
$$

\section{Properties of the method}

3.1. Order of the method

A linear difference operator of the linear multistep scheme (2.1) is defined as

$$
L\{y(x): h\}=y_{n+\frac{4}{5} \xi}-\sum_{i=0}^{1} \frac{\left(\frac{4}{5} \xi h\right)^{i}}{i !} y_{n}^{(i)}-h^{2} \sum_{i=0}^{5} \phi_{\xi i} f_{n+\frac{4}{5} i}, \quad \xi=1,2, \cdots, 5 .
$$

Expanding the terms in the difference operator (3.1) for each value of $\xi$ using Taylor series expansion, gives a family of expressions that can be generalized in the form given below individually

$$
L\{y(x): h\}=c_{0} y(x)+c_{1} h y^{\prime}(x)+c_{2} h^{2} y^{\prime \prime}(x)+\cdots+c_{p} h^{(p)} y(x)+c_{p+1} h^{(p+1)} y(x)+\cdots .
$$

A block method developed for solving second order differential equations is said to have order $p$ with its error constant obtained at $c_{p+2}$, where $c_{0}=c_{1}=c_{2}=\cdots=c_{p+1}=0$ and $c_{p+2} \neq 0$.

Therefore, adopting this linear difference operator to the block schemes (2.3), the order of the schemes are $(6,6,6,6,6)$ with error constants $\left(-\frac{101888}{73828125},-\frac{1245184}{369140625},-\frac{72192}{13671875},-\frac{524288}{73828125},-\frac{5632}{590625}\right)$.

\subsection{Zero-stability of the block method}

Normalizing the block schemes (2.3) to give the first characteristic polynomial as

$$
\rho(R)=\operatorname{det}\left(R A^{0}-A^{1}\right)=R^{4}(R-1),
$$

where

$$
A^{0}=\left(\begin{array}{lllll}
1 & 0 & 0 & 0 & 0 \\
0 & 1 & 0 & 0 & 0 \\
0 & 0 & 1 & 0 & 0 \\
0 & 0 & 0 & 1 & 0 \\
0 & 0 & 0 & 0 & 1
\end{array}\right), \quad A^{1}=\left(\begin{array}{ccccc}
0 & 0 & 0 & 0 & 1 \\
0 & 0 & 0 & 0 & 1 \\
0 & 0 & 0 & 0 & 1 \\
0 & 0 & 0 & 0 & 1 \\
0 & 0 & 0 & 0 & 1
\end{array}\right)
$$

The roots of $\rho(R)=0$ satisfy $\left|R_{j}\right| \leqslant 1, j=1, \cdots, 5$. Hence, the block method (2.3) is said to be zero-stable.

\subsection{Convergence of the block method}

A block method which is zero stable and has order greater than one is said to be convergent. Hence, the block schemes (2.3) satisfy these properties as shown in previous subsections and is therefore said to be convergent.

\subsection{Numerical experiments}

1. $y^{\prime \prime}=y^{\prime}, \quad y(0)=0, \quad y^{\prime}(0)=-1, \quad h=0.1$, $y=1-e^{x}$

2. $y^{\prime \prime}+\frac{6}{x} y^{\prime}+\frac{4}{x^{2}} y=0, \quad y(1)=1, \quad y^{\prime}(1)=1, h=\frac{0.1}{32}$, $y=\frac{5}{3 x}-\frac{2}{3 x^{4}}$.

Numerical experiments (1) and (2) are solved using the new order 6 hybrid block method and compared with the order 6 block method of [12] and the results are reported in Tables 1 and 2, respectively. Likewise, numerical experiment (2) was also solved by [5] with a block method of order 8 and the results is also reported in Table 2; 
3. $y^{\prime \prime}=x\left(y^{\prime}\right)^{2}, \quad y(0)=1, \quad y^{\prime}(0)=\frac{1}{2}, \quad h=\frac{1}{30}$, $y=1+\frac{1}{2} \ln \left(\frac{2+x}{2-x}\right)$.

Numerical experiment (3) was solved using the new order 6 hybrid block method and compared with the order 7 block method of [9]. The numerical results are reported in Table 3.

\section{Discussion of results}

In Tables 1 and 2, the hybrid block method was compared with [12] where the authors also presented an order six block method. However, despite both block methods being of the same order, the hybrid block method showed better stability in results and improved accuracy. Furthermore, in comparison to the numerical method by [5] having a higher order (order 8), more convergent results to the exact solution were also displayed by the hybrid block method as seen in Table 2. Table 3 finally shows another higher order method of order seven having less accuracy than the new block methods. This shows that the hybrid block method competes favourably with block methods of same order to itself or higher.

The convergence of the block method to the exact solution is displayed in figures. This is observed in the overlapping of the computed solution over the exact solution.

Table 1: Comparison of results for solving Numerical Experiment 1.

\begin{tabular}{lllll}
\hline$x$ & Exact Solution & Computed Solution & Error [12]; Order 6 & $\begin{array}{l}\text { Error (Hybrid Block } \\
\text { Method); Order 6 }\end{array}$ \\
\hline 0.08 & -0.083287067675 & -0.083287067692 & - & $1.734020 \mathrm{E}-11$ \\
0.10 & -0.105170918076 & - & $2.508826 \mathrm{E}-13$ & - \\
0.16 & -0.173510870992 & -0.173510871036 & - & $4.425317 \mathrm{E}-11$ \\
0.20 & -0.221402758160 & - & $6.493175 \mathrm{E}-11$ & - \\
0.24 & -0.271249150321 & -0.271249150394 & - & $7.218060 \mathrm{E}-11$ \\
0.30 & -0.349858807576 & - & $1.683146 \mathrm{E}-09$ & - \\
0.32 & -0.377127764336 & -0.377127764437 & - & $1.014698 \mathrm{E}-10$ \\
0.40 & -0.491824697641 & -0.491824697781 & $1.700635 \mathrm{E}-08$ & $1.406911 \mathrm{E}-10$ \\
0.48 & -0.616074402192 & -0.616074402420 & - & $2.268697 \mathrm{E}-10$ \\
0.50 & -0.648721270700 & - & $1.025454 \mathrm{E}-07$ & - \\
0.56 & -0.750672500296 & -0.750672500628 & - & $3.323523 \mathrm{E}-10$ \\
0.60 & -0.822118800391 & - & $2.558711 \mathrm{E}-06$ & - \\
0.64 & -0.896480879305 & -0.896480879750 & - & $4.447897 \mathrm{E}-10$ \\
0.70 & -1.013752707470 & - & $5.273300 \mathrm{E}-06$ & - \\
0.72 & -1.054433210644 & -1.054433211209 & - & $5.651533 \mathrm{E}-10$ \\
0.80 & -1.225540928492 & -1.225540929199 & $8.275935 \mathrm{E}-06$ & $7.067192 \mathrm{E}-10$ \\
0.88 & -1.410899706417 & -1.410899707342 & - & $9.252547 \mathrm{E}-10$ \\
0.90 & -1.459603111157 & - & $1.161667 \mathrm{E}-05$ & - \\
0.96 & -1.611696473423 & -1.611696474603 & - & $1.1800824 \mathrm{E}-09$ \\
1.00 & -1.718281828459 & - & $1.542187 \mathrm{E}-05$ & - \\
\hline
\end{tabular}

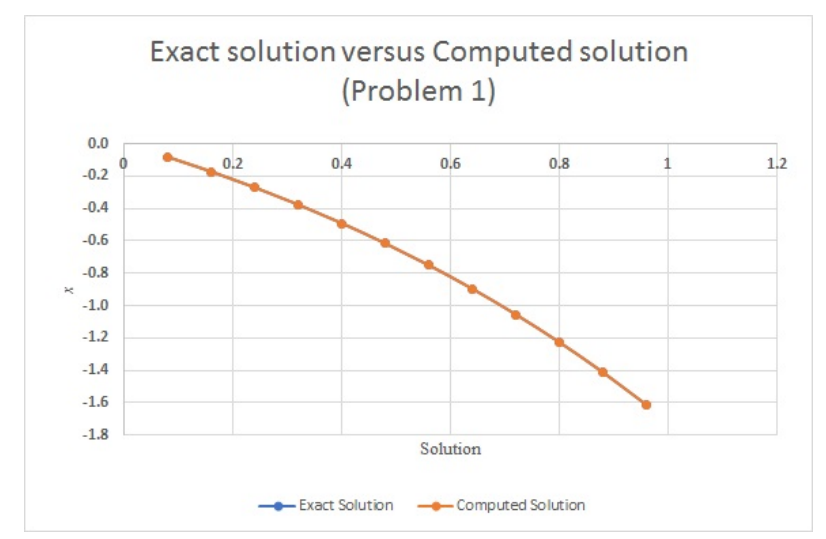

Figure 1: Exact solution and computed solution of Problem 1. 
Table 2: Comparison of results for solving Numerical Experiment 2.

\begin{tabular}{|c|c|c|c|c|c|}
\hline$x$ & Exact solution & Computed solution & Error [5]; Order 8 & Error [12]; Order 6 & $\begin{array}{l}\text { Error (Hybrid Block } \\
\text { Method); Order } 6\end{array}$ \\
\hline 1.002500 & 1.0024689314487823556 & 1.0024689314487823049 & - & - & 5.0700E-17 \\
\hline 1.003125 & 1.0030765258576962262 & - & 8.30000E-08 & $2.220446 \mathrm{E}-16$ & - \\
\hline 1.005000 & 1.0048764449022821194 & 1.0048764449022819961 & - & - & $1.2330 \mathrm{E}-17$ \\
\hline 1.006250 & 1.0060575030835162830 & - & 1.16000E-06 & 4.440892E-16 & - \\
\hline 1.007500 & 1.0072236041571325413 & 1.0072236041571323499 & - & - & $1.9140 \mathrm{E}-16$ \\
\hline 1.009375 & 1.0089449950888375792 & - & 6.63000E-06 & $1.110223 \mathrm{E}-15$ & - \\
\hline 1.010000 & 1.0095114535131059765 & 1.0095114535131057208 & - & - & $2.5570 \mathrm{E}-16$ \\
\hline 1.012500 & 1.0117410181679885289 & 1.0117410181679881877 & 9.49100E-06 & $1.998401 \mathrm{E}-15$ & 3.4120E-16 \\
\hline 1.015000 & 1.0139133046035961802 & 1.0139133046035956607 & - & - & 5.1950E-16 \\
\hline 1.015625 & 1.0144475426864138744 & - & 1.95350E-06 & 6.217249E-15 & - \\
\hline 1.017500 & 1.0160293009631512792 & 1.0160293009631505647 & - & - & 7.1450E-16 \\
\hline 1.018750 & 1.0170664942356726084 & - & $9.41600 \mathrm{E}-06$ & 8.659740E-15 & - \\
\hline 1.020000 & 1.0180899774202323528 & 1.0180899774202314488 & - & - & $9.0400 \mathrm{E}-16$ \\
\hline 1.021875 & 1.0195997547562875920 & - & 4.65050E-05 & - & - \\
\hline 1.022500 & 1.0200962865395044606 & 1.0200962865395033730 & - & - & $1.0876 \mathrm{E}-15$ \\
\hline 1.025000 & 1.0220491636294317413 & 1.0220491636294304526 & 4.71220E-05 & $6.883383 \mathrm{E}-15$ & $1.2887 \mathrm{E}-15$ \\
\hline 1.027500 & 1.0239495270871683888 & 1.0239495270871668216 & - & - & $1.5672 \mathrm{E}-15$ \\
\hline 1.028125 & 1.0244165187384026804 & - & $1.86926 \mathrm{E}-04$ & 7.638334E-14 & - \\
\hline 1.030000 & 1.0257982787358190470 & 1.0257982787358171884 & - & - & $1.8586 \mathrm{E}-15$ \\
\hline 1.031250 & 1.0267035775008059839 & - & $4.43321 \mathrm{E}-04$ & $6.461498 \mathrm{E}-14$ & - \\
\hline
\end{tabular}

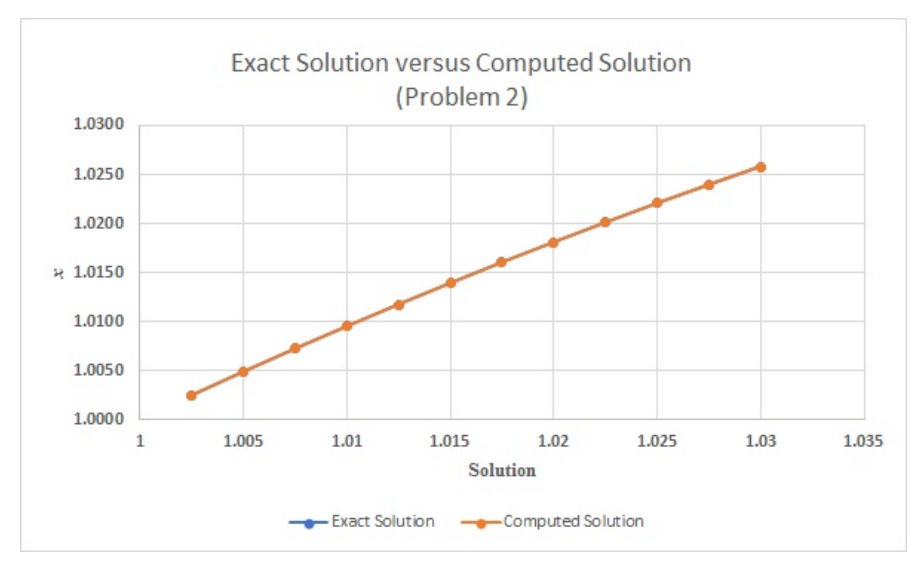

Figure 2: Exact solution and computed solution of Problem 2.

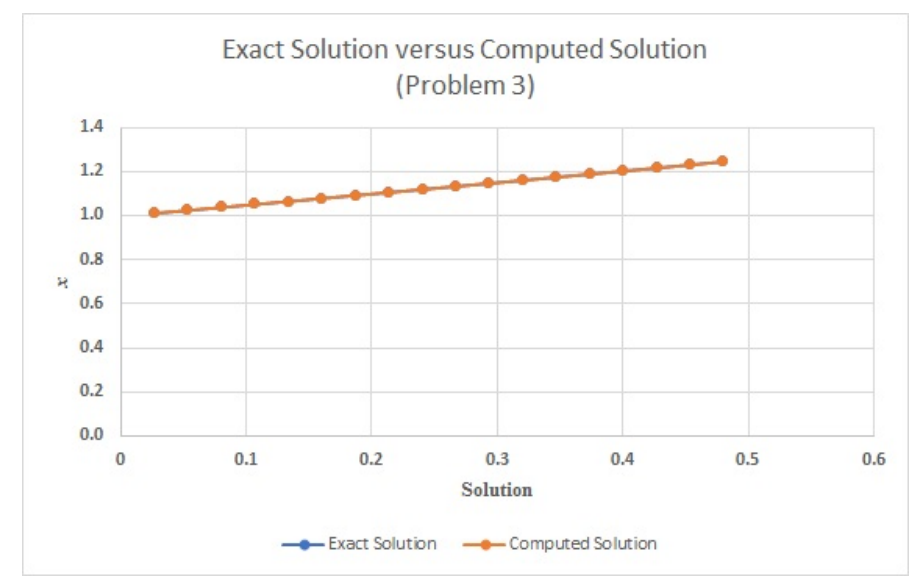

Figure 3: Exact solution and computed solution of Problem 3. 
Table 3: Comparison of results for solving Numerical Experiment 3.

\begin{tabular}{lllll}
\hline$x$ & Exact solution & Computed solution & Error [9]; Order 7 & $\begin{array}{l}\text { Error (Hybrid Block } \\
\text { Method); Order 6 }\end{array}$ \\
\hline 0.0267 & 1.0133341235410806625 & 1.0133341235410905060 & - & $9.843500 \mathrm{E}-15$ \\
0.0300 & 1.0166682101337958000 & - & $1.865175 \mathrm{E}-14$ & - \\
0.0533 & 1.0266729903526463600 & 1.0266729903526703244 & - & $2.396440 \mathrm{E}-14$ \\
0.0800 & 1.0400213538367682129 & 1.0400213538368057585 & - & $3.754560 \mathrm{E}-14$ \\
0.1000 & 1.0500417292784914000 & - & $1.445510 \mathrm{E}-13$ & - \\
0.1067 & 1.0533839877128530364 & 1.0533839877129033472 & - & $5.031080 \mathrm{E}-14$ \\
0.1333 & 1.0667656963122613116 & 1.0667656963123301728 & - & $6.886120 \mathrm{E}-14$ \\
0.1600 & 1.0801713250375896917 & 1.0801713250377265533 & - & $1.368616 \mathrm{E}-13$ \\
0.1867 & 1.0936057710440732071 & 1.0936057710442945501 & - & $2.213430 \mathrm{E}-13$ \\
0.2000 & 1.1003353477310756000 & - & $3.779332 \mathrm{E}-11$ & - \\
0.2133 & 1.1070739942028159319 & 1.1070739942031199546 & - & $3.040227 \mathrm{E}-13$ \\
0.2400 & 1.1205810284084440352 & 1.1205810284088286229 & - & $3.845877 \mathrm{E}-13$ \\
0.2667 & 1.1341319932973396721 & 1.1341319932978235297 & - & $4.838576 \mathrm{E}-13$ \\
0.2933 & 1.1477321064469179382 & 1.1477321064476226767 & - & $7.047385 \mathrm{E}-13$ \\
0.3000 & 1.1511404359364668000 & - & $3.428134 \mathrm{E}-08$ & - \\
0.3200 & 1.1613866961315255154 & 1.1613866961324884439 & - & $9.629285 \mathrm{E}-13$ \\
0.3467 & 1.1751012147165574585 & 1.1751012147177759154 & - & $1.218457 \mathrm{E}-12$ \\
0.3733 & 1.1888812527794142948 & 1.1888812527808852324 & - & $1.470938 \mathrm{E}-12$ \\
0.4000 & 1.2027325540540821910 & 1.2027325540558470662 & $6.987109 \mathrm{E}-08$ & $1.764875 \mathrm{E}-12$ \\
0.4267 & 1.2166610313055652953 & 1.2166610313078790954 & - & $2.313800 \mathrm{E}-12$ \\
0.4533 & 1.2306727832513104865 & 1.2306727832542528585 & - & $2.942372 \mathrm{E}-12$ \\
0.4800 & 1.2447741126593528930 & 1.2447741126629217090 & - & $2.942372 \mathrm{E}-12$ \\
0.5000 & 1.2554128118829952000 & - & $2.017066 \mathrm{E}-07$ & - \\
\hline
\end{tabular}

\section{Conclusion}

This article has presented a new hybrid block method of order six for numerically approximating the solution of second order initial value problems. This hybrid block method has satisfied possessing properties that will confirm its convergence when applied to solve second order ODEs. This is seen in the numerical results shown above, where the hybrid block method shows a good level of convergence and likewise performing better than previously existing methods in literature. Hence, authors can conveniently utilize this hybrid block method when solving second order IVPs. Also, the new approach shown in this article for developing this method which from observation is seen to be less cumbersome than previously existing approach is also a new contribution to authors considering developing hybrid block methods.

\section{Acknowledgment}

This work is funded by Universiti Utara Malaysia.

\section{References}

[1] A. O. Adesanya, Block methods for direct solutions of general higher order initial value problems of ordinary differential equations, PhD Thesis, Federal University of Technology, Akure, Nigeria, (2011). 1

[2] A. O. Adesanya, M. R. Odekunle, A. O. Adeyeye, Continuous block hybrid predictor corrector method for the solution of $y^{\prime \prime}=f\left(x, y, y^{\prime}\right)$, Int. J. Math. Soft Comput., 2 (2012), 35-42. 1

[3] A. O. Adesanya, D. M. Udoh, A. M. Ajileye, A new hybrid block method for the solution of general third order initial value problems of ordinary differential equations, Int. J. Pure Appl. Math., 86 (2013), 365-375. 1

[4] T. A. Anake, D. O. Awoyemi, A. O. Adesanya, One-step implicit hybrid block method for the direct solution of general second order ordinary differential equations, IAENG Int. J. Appl. Math., 42 (2012), 224-228. 1

[5] A. M. Badmus, A new eighth order implicit block algorithms for the direct solution of second order ordinary differential equations, Amer. J. Comput. Math., 4 (2014), 376-386. 2, 4, 2

[6] A. A. James, O. A. Adesanya, K. M. Fasasi, Starting order seven method accurately for the solution of first initial value problems of first order ordinary differential equations, Progress Appl. Math., 6 (2013), 30-39. 1

[7] S. J. Kayode, O. Adeyeye, A 3-step hybrid method for direct solution of second order initial value problems, Aust. J. Basic Appl. Sci., 5 (2011), 2121-2126. 1 
[8] S. J. Kayode, O. Adeyeye, Two-step two-point hybrid methods for general second order differential equations, Afr. J. Math. Comput. Sci. Res., 6 (2013), 191-196. 1

[9] J. O. Kuboye, Z. Omar, Derivation of a six-step block method for direct solution of second order ordinary differential equations, Math. Comput. Appl., 20 (2015), 151-159. 1, 3, 3

[10] Z. A. Majid, N. Z. Mokhtar, M. Suleiman, Direct two-point block one-step method for solving general second-order ordinary differential equations, Math. Probl. Eng., 2012 (2012), 16 pages. 1

[11] R. I. Okuonghae, M. N. O. Ikhile, A class of hybrid linear multistep methods with A( $\alpha$ )-stability properties for stiff IVPs in ODEs, J. Numer. Math., 21 (2013), 157-172. 1

[12] Z. Omar, J. O. Kuboye, A new implicit block method for solving second order ordinary differential equations directly, G.U. Journal of Science, 28 (2015), 689-694. 2, 4, 1, 2

[13] P. S. Phang, Z. A. Majid, M. Suleiman, Solving nonlinear two point boundary value problem using two step direct method, J. Qual. Measure. Anal., 7 (2011), 129-140. 1

[14] K. Rauf, S. A. Aniki, S. Ibrahim, J. O. Omolehin, A zero-stable block method for the solution of third order ordinary differential equations, Pac. J. Sci. Tech., 16 (2015), 91-103. 1 